\title{
Clinical pathways of metastatic spinal cord compression: Orthopedics experience based on hospital admissions
}

\author{
Sharifa Ezat Wan Puteh ${ }^{1 *}$, Noraziani Khamis ${ }^{1}$ and Sabarul Afian Mokhtar ${ }^{2}$ \\ ${ }^{1}$ Department of Community Health, Faculty of Medicine, Universiti Kebangsaan Malaysia Medical Centre, Kuala Lumpur, Malaysia \\ ${ }^{2}$ Department of Orthopaedic and Traumatology, Faculty of Medicine, Universiti Kebangsaan Malaysia Medical Centre, Kuala Lumpur, Malaysia
}

\begin{abstract}
Bone metastases are more common than primary bone cancer. Metastatic bone event of spinal cord compression causes significant morbidity and advances in medical approaches improve management of bone events. Morbidity or healthcare resource utilization could impose an increased pressure on healthcare system. Objective of study is to determine patterns of clinical activities in managing metastatic spinal cord compression secondary to primary breast and prostate cancer patients admitted to orthopedics unit in public teaching hospital. Findings were described into clinical pathways. Within year 2013, majority of patients presented with MSCC had primary breast cancer (60\%), Malay ethnicity (70\%), presented with neurological deficits (60\%), were able to mobilize at presentation (60\%), with poorer prognosis (60\%), and have pre-existing cancer diagnosis (70\%). As many as $583(19 \%)$ numbers of cases screened to have problem related to spine from the ward census. There are two main arms of clinical pathways designed from the data which are surgery and non surgery interventions. Approximately $67 \%$ of cases had radiotherapy and remaining 33\% received best supportive care within those $60 \%$ of 'poor prognosis' arm. Four patients (40\%) with 'moderate to good prognosis' arm had surgery followed by radiotherapy. Posterior decompression surgery performed to all patients fits for surgery. Given the increasing incidence of metastatic disease along with evolving treatment options, research related to treatment algorithms can be suggested to further optimize patient outcomes. The essential steps in treating patients with a specific clinical problem of MSCC can be translated into a multidisciplinary care plans known as clinical pathway. The pathways support experts' deciding on allocation of activities and costs incurred in managing MSCC. Findings supports subsequent other economics related study.
\end{abstract}

\section{Introduction}

Managing metastatic spine cord compression (MSCC) is becoming increasingly frequent for clinicians and surgeons. New improved oncology treatments result in a relatively longer cancer patients' survival. Bone is the third most common body system prone to metastases after liver and lung with spine serves as the most common site of skeletal metastases [1-5]. Primary cancer of spine is rare, but the spine is generously supplied with blood and highly susceptible to metastatic deposits [6-7].

Spinal metastases commonly arise from three primary cancer sites of breast (21\%), lung (14\%), prostate (8\%), followed by renal, gastrointestinal, thyroid, sarcoma, lymphomas, and multiple myelomas [7-10]. More than $10 \%$ of cancer patients develop symptoms secondary to spinal disease, $40 \%$ to $70 \%$ of those cases will involve several vertebral levels and certain proportion of spinal metastases leads to neurologic compromise [11,12]. The proportion of cancer patients affected by spinal metastases, spinal cord compression and the type of spine involved are as illustrated by Klimo et al. [13]. The thoracic (70\%) and lumbar spine (20\%) are the most common site involved [13].

Spinal cord compression occurs mainly secondary to degenerative disease, or spinal metastases which can result in considerable morbidity. Cancer patients presented with sign and symptoms of spinal metastases require expeditious treatment in hospital. Careful monitoring is required for gradual change in clinical presentation to allow optimal decision making, on the other hand, emergency surgical intervention can be a must in obvious rapid deterioration circumstances [14]. A clinical pathway is also known as integrated care pathway, coordinated care pathways, care pathways, critical pathways, and care maps, described in many literatures. Clinical pathways are a concept to introduce patient-focused care [15]. Patterns of clinical activities in managing MSCC secondary to primary breast and prostate cancer patients admitted to orthopedic units in a public teaching hospital was determined. Findings were translated into clinical pathways which can be used for subsequent health economics related study. Later, the pathways support experts' deciding on allocation of activities and costs incurred in managing MSCC.

\section{Methods}

All 3,141 numbers of admissions cases to orthopedics units in public tertiary teaching hospital within year 2013 documented in wards census were reviewed. The census includes information on reason for admission and diagnosis prior to discharge for all cases monitored in wards. The patients' identification was shortlisted if they are at least 18 years old and admitted with problem or disease related to spine. Then, each discharged summaries documents, and patients' medical records of the listed cases were retrospectively reviewed to verify their reasons of admission and discharge from orthopedics wards together with information on diagnosis of primary and secondary cancer. Patients

Correspondence to: Sharifa Ezat Wan Puteh, MD, MPH, PhD, Department of Community Health, Faculty of Medicine, University Kebangsaan, Malaysia Medical Centre, Kuala Lumpur, Malaysia, Tel: +603-9145 5887, Fax: +603-9173 7825;E-mail: sh_ezat@yahoo.com

Keywords: spinal cord compression, spine metastases, breast cancer, prostate cancer, clinical pathway

Received: June 22, 2015; Accepted: July 30, 2015; Published: August 03, 2015 
diagnosed clinically with metastatic spine cord compression present either as spinal metastases with known metastatic disease, with a history of cancer without known metastases, or as a patient with no known history of cancer were considered for study. Only each patient with diagnosis of a primary breast (female only) or prostate cancer having underlying metastatic cord compression was eligible for inclusion in this study. Cases were excluded from the lists if diagnosis does not fulfill the criteria. The lists of cases are further verified with orthopedic spine consultant for their diagnosis, prognosis and treatment care received.

For each patient, the following data were extracted: gender, age, clinical presentation, procedures done to the spine, human resources involved for the procedure to spine, total cost for equipments or implants for spine, whether special care unit needed after the procedure with its duration of stay, and total duration of hospital stay for the presentation. Surgical procedures among patients with terminally ill metastatic cancer might cause patients to undergo special care for recovery. The description of each case was for their episode of admission for clinical diagnosis of metastatic spinal cord compression.

Objectives of the review from the census was to prepare a list of orthopedics cases admitted to public teaching hospital in year 2013 with possibility having underlying metastatic spinal cord compression secondary to breast or prostate cancers. Subsequently, the patterns of clinical activities in managing spinal cord compression secondary to spinal metastases were determined. Findings of this review were applied to develop clinical pathways for managing metastatic spinal cord compression based on one year hospital admissions.

The clinical pathways are in widespread use and act as one systematic way to organized and follow-up patients focused care processes in a predefined setting. Care pathways can be used locally to direct and plan activity within hospitals even though in reality, patients require individualized care, rapid change of needs and can present with a number of problems. Considering the complexity of interaction between patients and the need of different treatment modality along the patients' life cycle, we develop pathways with some assumption. The assumptions are made to ease the illustration of pathways for their treatment plan. Management of treatment comprises of activities at first time of meeting each patient attending specialist centers in our hospital. The percentages allocated within each activity are based on proportion of $100 \%$ patients seen at one time in hospital within one year. Therefore the pathways will explain the possible ways that a group of metastatic breast or prostate cancer patients would pave the way within one year period.

\section{Results}

There were $583(18.6 \%)$ numbers of cases having problem or disease related to spine, shortlisted from the census. A potential sample of 18 cases $(3.1 \%)$ having problem related to spine with underlying primary breast or prostate cancers was initially generated out of 583 cases admitted within a year. However, 6 out of 18 cases (33.3\%) were excluded because spinal metastases were not diagnosed. The final number of cases sampled that fulfilled the criteria of search was 10 in which previous 2 cases were further excluded because their cord compression episodes were not secondary to spinal metastases.

\section{Characteristic of patients}

Patients presented with MSCC were more likely to have primary breast cancer $(60 \%)$, Malay ethnicity $(70 \%)$, presented with some degree of neurological deficits $(60 \%)$, were able to mobilize at presentation $(60 \%)$, having poorer prognosis $(60 \%)$, and had preexisting cancer diagnosis (70\%). There were 6 out of 10 cases had breast cancer with spine metastases (60\%) and 4 out of 10 cases had prostate cancer with spine metastases (40\%). Those cases with presence of underlying spine metastases were managed clinically as metastatic spinal cord compression. Patients' age ranges from 45 to 77 years old. All 5 breast cancer patients aged less 65 years old (50\%) however, only one breast cancer with 4 prostate cancers were in another $50 \%$ of cases grouped into age 65 years old and above (Table 1). About $30 \%(n=3)$ of cases had no pre-existing cancer diagnosis and $60 \%(n=6)$ of patients have history of able to mobilize (ranges from walk independent to with assistance) until their symptoms and/or neurological damage are presented to the hospital in 2013. Among 10 cases with MSCC, as many as $40 \%$, comprising of $(n=3)$ of breast cancer and $(n=1)$ of prostate cancer classified as having 'moderate to good prognosis'. The remaining $60 \%$, which were made up of 3 breast cancer and 3 prostate cancer cases were with 'poor prognosis' group. About half of the cases with 'moderate to good prognosis' group presented with some forms of neurological function deficits.

\section{Treatment choice}

The data extracted from the records were illustrated in the clinical algorithm (Figure 1). As per initial treatment at presentation, about $67 \%(n=4)$ had radiotherapy and $33 \%(n=2)$ receives best supportive care, among the $60 \%(\mathrm{n}=6)$ of 'poor prognosis' arm. Rarely surgery interventions were used in isolation. Four cases (40\%) with 'moderate to good prognosis' arm had surgery followed by radiotherapy.

Several intervention options exist for the MSCC, which include medical treatments of chemotherapy or steroids, radiotherapy, and surgery. In general, patients with MSCC proceed to either surgical or non surgical procedures in their first clinical presentation. As much as $50 \%(n=3)$ of breast cancers and $25 \%(n=1)$ of prostate cancer cases diagnosed clinically with metastatic spinal cord compression were fit to go through surgical procedures to spine. Regardless of underlying neurological status, every single case needed radiotherapy post operatively within a year. None of the cases were identified to be under 'preoperative radiotherapy' or 'surgery only to spine' arms

Table 1. Characteristic of patients with metastatic cord compression within each type of primary cancer.

\begin{tabular}{|c|c|c|c|}
\hline \multirow{2}{*}{\multicolumn{2}{|c|}{ Classification }} & \multicolumn{2}{|c|}{$\begin{array}{c}\text { Cases with Spinal Cord } \\
\begin{array}{c}\text { Compression Within Each Type } \\
\text { of Cancer } n(\%)\end{array}\end{array}$} \\
\hline & & \multirow[t]{2}{*}{$\begin{array}{l}\text { Breast } \\
\text { cancer }\end{array}$} & \multirow[t]{2}{*}{ Prostate cancer } \\
\hline Gender & & & \\
\hline & Male & 0 & $4(100)$ \\
\hline & Female & $6(100)$ & 0 \\
\hline \multicolumn{4}{|l|}{ Age } \\
\hline & less 65 years old & $5(83)$ & 0 \\
\hline & More 65 years old & $1(17)$ & $4(100)$ \\
\hline \multicolumn{4}{|l|}{ Ethnic } \\
\hline & Malay & $6(100)$ & $1(25)$ \\
\hline & Non Malay & 0 & $3(75)$ \\
\hline \multicolumn{4}{|c|}{ Neurological status } \\
\hline & Neurological Function Intact & $3(50)$ & $1(25)$ \\
\hline & Neurological Function deficits & $3(50)$ & $3(75)$ \\
\hline \multicolumn{4}{|c|}{ Prognosis } \\
\hline & Moderate-Good & $3(50)$ & $1(25)$ \\
\hline & Poor & $3(50)$ & $3(75)$ \\
\hline
\end{tabular}




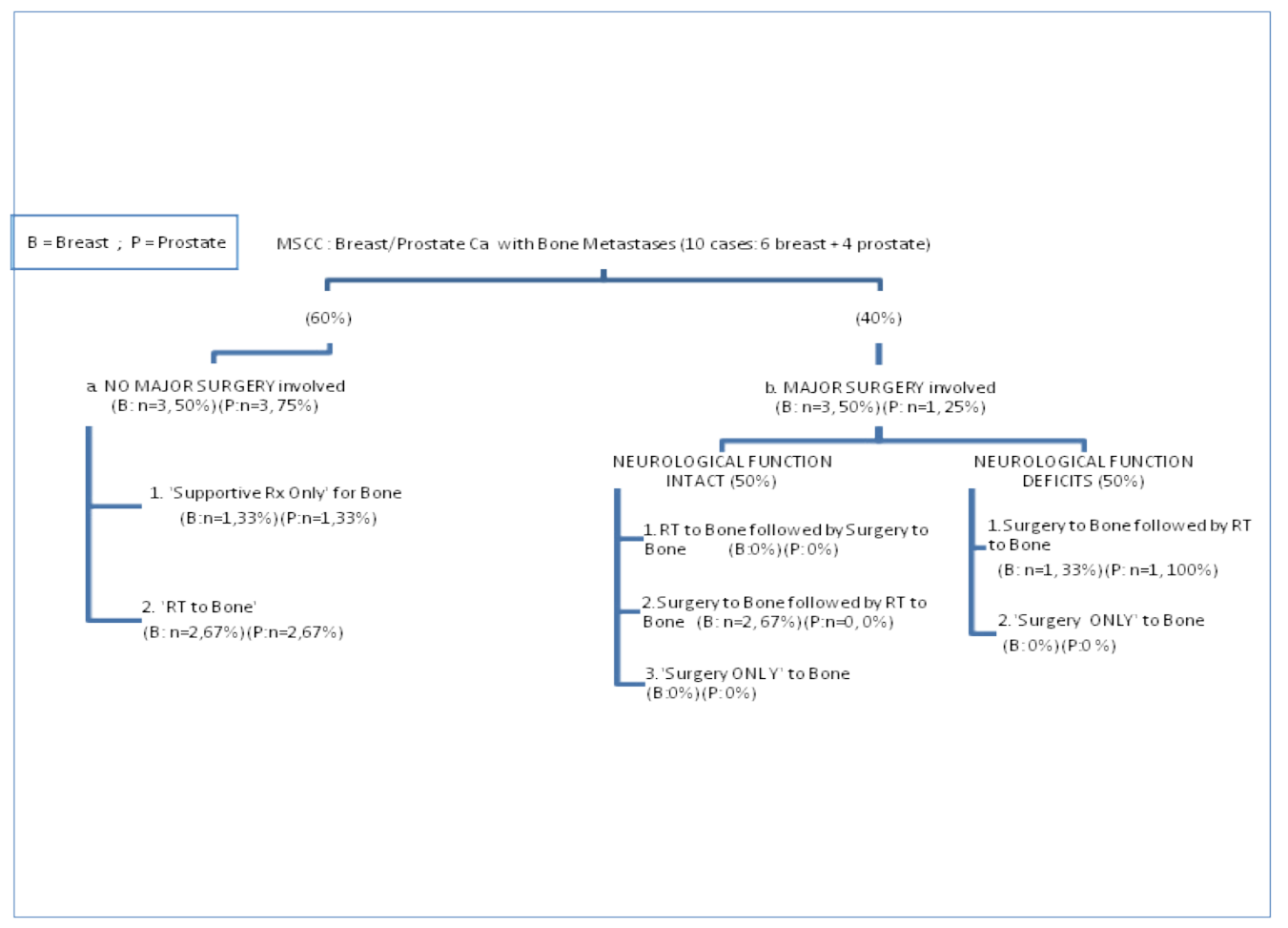

Figure 1. Distribution of patients within each types of cancer.

of treatment choice. Cases with suspicion of MSCC were admitted to ward and laid flat in bed. They received mobilization nursing and vital signs were monitored using neurosurgical observation chart for at least 1-2 days in wards. Intravenous corticosteroid was administered to $50 \%$ of patients until further definitive treatment. Gradually the dose was reduced however some cases experience an increase in dosage if neurological functions deteriorate. Current clinical pathway adopt a minimum dosage of dexamethasone $10 \mathrm{mg}$ daily dose for two days prior to treatment, and followed by $4 \mathrm{mg}$ daily dose for five days, subsequently $2 \mathrm{mg}$ daily dose for two weeks. Almost all cases with symptoms suggestive of spinal metastases or having neurological symptoms or signs suggestive of MSCC had undergone CT scan and MRI.

\section{Surgery}

About $67 \%(n=2)$ out of breast cancer cases undergone surgery, required 'embolization' procedure prior to surgery. A common minimum of human resources needed for the procedure were identified. The cost of catheter for embolism, and embolization agent were approximately between RM 3,000 - 5,000.00 (in Malaysian Ringgit). Patients decided for surgical intervention underwent 'Posterior Decompression Surgery' under general anesthesia. Total duration of surgical procedure ranged from 1 hour 35 minutes to 3 hour 30 minutes; and 1 hour 35 minutes to 3 hour 29 minutes for respective breast and prostate cancer patients. The breakdowns of costs related to prosthesis ranged from RM 19,932.00 to RM 48,230.00 for breast cancer; and RM 15,424.00 for prostate cancer cases. Each of the $67 \%$ of breast cancer cases required a one day admission in intensive care unit, after the surgical procedure. Investigation of X-rays and blood counts were done post operatively. There were more breast (50\%) compared to prostate cancer cases (25\%) undergone surgical procedure to spine.
Analgesics were prescribed until their diseases were under control with other systemic therapies.

Among those under surgical arm, their total length of hospital stays ranged from one to two weeks for breast cancer cases, and three weeks for prostate cancer. The breast cancer cases with intact neurological function stay shorter duration (range 10-62 days), than breast cancer with neurological deficits (range 14-63 days). Wound Inspection was done in wards at third day post operation, and removal of stitch at two weeks post operatively. The cases with neurological deficits have radiotherapy while in wards but those with intact neurological function undergone radiotherapy as outpatients at two weeks post operatively. Only one prostate case died after 20 days of admission.

\section{Non surgery procedure}

Pathway of cases with poorer prognosis is differentiated into a non surgical procedure of 'palliative radiotherapy'; or 'supportive treatment only' as their therapy at first presentation (Figure 1). The cases were either too frail or unfit for surgery. The breast cancer cases account for $50 \%(n=3)$ and prostate cancer cases account for $75 \%(n=3)$ of total sample. From the proportion of these patients, $67 \%(n=2)$ of the cases from each cancer type undergo palliative radiotherapy 20 Gy 5 fractions. A common minimum dosage of analgesics and medicines prescribed were identified. Total length of hospital stays ranged from one to two weeks for breast cancer cases, and two to three weeks for prostate cancer.

The remaining 33\% $(n=1)$ within each cancer types received 'supportive therapy only' as their initial treatment. Management for 'Supportive therapy only' for the clinical pathways are defined as palliative care excluding chemotherapy, radiotherapy or surgery. Among those in 'supportive therapy only', almost all breast cancer 
cases receive Vetebroplasty or Khyphoplasty and majority of prostate cancer patients received 'External Spinal Support' such as a custom made brace. The external spinal support is considered for patients with severe mechanical pain and/or imaging evidence of spinal instability, who are unsuitable for surgery. One set of 'Thoracolumbar Spine Orthotics' costs about RM 500.00 to RM 700.00. Patients decided for Vetebroplasty under general anesthesia require a total duration of one hour of surgical procedure. Vetebroplasty equipment costs about RM 20,780.00 in average. The case had a one day of intensive care unit admission after Vetebroplasty. Their total length of hospital stays ranged from nine weeks for breast cancer cases, and two weeks for prostate cancer.

\section{Complication of MSCC}

Roughly $50 \%$ of both types of cancer cases suffer complication secondary to MSCC upon admission to orthopedics unit. Within a year, there were more prostate cancer $n=2(50 \%)$ presented with bladder incontinent compared to breast cancer $n=1(16.7 \%)$ on their admission. More prostate cancer $n=2(50 \%)$ presented with bowel incontinent as their first presentation of complication comparing with breast cancer $n=2(33 \%)$. In general, bladder dysfunction was managed with a urinary catheter on free drainage. No other complication documented within the episode of admission.

\section{Discussion}

The indications for different treatment modality were reflection of selection by treating physicians in our hospital. Surgeons were assisted by scoring systems, such as Tokuhashi, Tomita, in selecting the type of invasiveness of surgical procedure to be performed to cases with a reasonable life expectancy [16]. Surgery is preferred in the presence of spinal instability and neurological signs in patients who have a 'moderate to good' prognosis. Abrahm [17] in his review emphasized that patients should be explained for clear understanding of treatment and desired outcomes on treatment options. These include the pros and cons of each treatment choice available together with expected chance of maintaining or regaining ambulation after treatment [17].

Radiation therapy had evolved from being a gold-standard in the treatment of spinal cord compression from metastatic cancer, to benefiting patients with radiotherapy serving an adjuvant role [18]. In view of attempt to determine most efficacious treatment options, Kaloostian et al. [19] had suggested algorithm for management of breast and prostate patients with metastatic spinal disease to include surgery and surgery plus postoperative radiotherapy. Surgery plus postoperative radiotherapy could benefit breast and prostate patients suffering from symptomatic spinal cord compression at a single site rather than at multiple sites [19]. Almost none of our cases within a year had required 'preoperative radiotherapy' or 'surgery only to spine' at their initial presentation of MSCC.

Corticosteroid is indicated in spinal cord compression [20]. Steroids initiated to reduce edema and prevent further injury to the cord. Those on steroid have random blood sugar monitored for side effect of glucose secondary to steroids. Corticosteroids are best used in short term at the lowest effective dose. The risk of steroid withdrawal was reduced by tapering dose in case of discontinuing corticosteroids used for longer than two weeks $[21,22]$.

The cases with symptoms suggestive of spinal metastases or having neurological symptoms or signs suggestive of MSCC undergo CT scan for staging or restaging and help in operative planning purposes [23].
MRI is the gold-standard investigation investigates the extent of soft tissue and bone involvement in MSCC for patients going for surgery [28]. MRI should be done early so that definite treatment can be planned within a week [9].

Generally, presentation of MSCC varies from obvious instability with clear clinical deformity and associated pain; to more subtle instability without overt clinical symptoms [23]. Spinal cord compression requires urgent referral and diagnosis by having a high clinical suspicion and with a low threshold to initiate investigation and treatment in order to maintain neurological function $[24,25]$. The importance of timing of surgery as an important factor contributing to the likely neurological outcome is as outlined in the NICE guidelines [9]. Ideally, those with spinal cord compression should undergo surgery within 48 hours of onset of neurologic symptoms because delayed surgical intervention associates with worse neurological outcomes [14]. However, the timing of surgery might not influence length of hospital stay, complication rate or patient survival [14].

Prostate has fewer tendencies to bleed as they have more osteoblastic or sclerotic type of bone as compared to breast cancer with bone metastases [26]. Preoperative embolization procedure of spinal metastases is effective in restricting preoperative perfusion and intraoperative blood loss can be significantly reduced after embolization [27].

Location of the cancer on metastatic spine often determines surgical approaches [28]. Reasons for selecting different approaches vary and it may be guided by factors like anatomy of the disease or patient-related factor [29]. The anterior and posterior approaches used for cancer in thoracic and lumbar spine allowed adequate access for decompression for instance.

The end of life for patients with MSCC is 'rewarding' to patients and healthcare providers if interventions used in a timely manner despite of challenging and costly management of MSCC [16]. Conceding that surgery in spinal metastases mainly not for curative, the goals of surgery are for symptomatic pain relief, restoring structural stability to the spine and preventing or reversing neurological compromise without causing excessive morbidity or without undue risk to the patient $[30,31]$. Surgery is offered for spinal cord decompression and to achieve spinal column stability, either by instrumentation or vertebral body reinforcement; and to maximize the probability of preserving spinal cord function. The potential for immediate and prolonged improvement in pain, function and quality of life in patients with symptomatic vertebral metastases should be considered during the decision making process [31]. Spinal fixation is needed if the spine is considered unstable within the lifetime of the patient either after a planned decompression, or if it is predicted that tumor progression will lead to spinal instability [16]. The procedure includes a minimum possible number of spinal segments are fixed for adequate spinal stability. The instrumentation is applied to spine despite of bone fusion might not occurring. The mechanical properties of the implant used to treat the pathological fracture should be durable enough to withstand along the duration of patient's survival time. Furthermore only onethird to one-fifth of pathological fractures unites, despite adjuvant therapy [26].

Pathological fracture is common among MSCC with the incidence ranges from $30 \%$ to $40 \%$ in literatures. Vertebral body compression fractures would lead to increased pain, neurological impairment, and reduction of quality of life [32]. Osteolytic lesions are most at risk of pathological fractures, and breast cancer causes $50 \%$ of pathological 
fractures that are due to metastases [18]. A retrospective review of 162 patients undergoing decompressive surgery for metastatic epidural spinal cord compression (MESCC) at an academic tertiary care institution from 1995 to 2007 showed factors that independently predicted preoperative vertebral pathological fractures includes primary breast cancer [32].

Chemotherapy and radiotherapy generally are preferable treatment modalities and surgery can be a last resort performed for older patients. A retrospectively reviewed of patients aged more 60 years who had surgery for spinal metastases within year 2000 to 2010 showed that pain relief, neurologic improvement, and restoration of general condition can be achieved with surgery. However, the surgery was with high risk of complications [33]. The results of systematic review from 1970 to 2007 suggest that patients treated with surgical decompression and instrumented stabilization for MSCC had a greater chance of decreased pain and improved ambulatory function compared with patients who received radiation alone [34].

Early and appropriate pain control while patients were being assessed or investigated and treated are essential step of care and this can be achieved by prescribing a combination of analgesics. Syed et al. [35] compared two radiotherapy groups (either single 8 Gy fractions or 5 fractions of 4 Gy) had shown a consistent findings with other study where palliative radiation showed a reduction in analgesic consumption at two months post radiation. Nevertheless, variation in socioeconomic status or healthcare policy could influence the practice of prescribing or availability of the analgesics in some part of world [35]. Local palliative care could be a more appropriate treatment plan for a patient who is too frail for treatment [36].

In general, radiotherapy is more commonly utilized for treatment than surgery for prostate elderly patients with MESCC [37]. A large population-based study showed $4.8 \%$ of 14,800 stage IV prostate cancer between years 1991 to 2007 developed MESCC. About 50\% of the patients underwent radiotherapy, while $15 \%$ underwent spinal surgery with or without radiotherapy. Older patients with spinal cord compression and those with comorbidities were less likely to be treated with either form of treatment in this palliative care setting. Spencer et al. [37] commented on randomized trial by Patchell et al. study [38] had sampled younger age patients with a variety types of cancers leading to MESCC, therefore, the findings were less applicable to older patients with only advanced prostate cancer.

Untreated patients with cord compression will invariably progress in morbidity. Spending more time in the hospital for symptom relief generally can be more costly. Nevertheless, surgery and radiotherapy are palliative therapies which can be a significant end-of-life consideration for patients. For example, patients treated only with a $20 \mathrm{~Gy}$ dose in five fractions were evaluated for its impact on quality of life. The evaluation was at three different times: before radiotherapy, after 30 days and 6 months, between year 2008 to 2010 . Their final pain score value was $25 \%$ lower than initial analysis and significant improvement noted in functional capability and social aspects categories [39].-

Spencer et al. [37] discussed on an additional days staying in hospital among patients who received either radiotherapy and/or surgery. For instance, there was a trend toward longer length of stay for those with 2 or more comorbidities who had surgery \pm radiation. Their results shown those underwent radiotherapy or surgery spent an additional 11 and 29 days, respectively and an additional 10 days for either group noted with other trial comprises relatively younger samples [38].
Our findings revealed that patients presented with some form of neurological deficits and they received radiotherapy while in wards. Radiotherapy relieves compression of the spine and nerve roots by causing cell death in the rapidly dividing tumor tissue hence effectively providing pain relief; and improving or the neurological deficit [9]. A wide range of published response rates to radiotherapy of painful bone metastases ranges from $60 \%$ to $90 \%$, partly relates to the subjective nature of mechanism of pain in spine metastases. A more complex pathophysiological mechanism of pain could also be underlying spinal metastatic disease which is different from pain by bone metastases at other sites [40]. Nevertheless, the systemic therapies (chemotherapy, hormone therapy, together with steroids and concomitant use of analgesics) could be confounders that may influence the response rates to radiotherapy aimed to address pain relief [40]. Majority of prostate cancer patients in our study received non surgical palliative care plan as their initial treatment plan instead of surgery. Generally, an external spinal support is considered for patient with severe mechanical pain and/or imaging evidence of spinal instability, who are unsuitable for surgery. A newer surgical treatment options include of minimally invasive techniques such as kyphoplasty or vertebroplasty [28]. Surgical treatments could also involve simple spinal decompression, spinal stabilization procedures, or both, where vertebroplasty or kyphoplasty is to be considered for pain relief and to prevent further collapse of the vertebrae [23]. Increasing the patients' remaining quality of life is aimed for patients not eligible for surgery because of their short life expectancy [41].

Findings of our study showed only neurological complication were presence during the episode of admission. Prostate cancer cases presented more in proportion for this as compared to breast cancer. Kim et al. [34] in a review shown an overall complication rate of $29 \%$ (ranged between 5\%-65\%), and the rate of mortality of 5\% (ranged between: $0 \%-22 \%$ ) within 30 days after surgery compared with patients who received radiation alone shown. However, the review were limited as only a few studies documented systemic disease progression during therapy and also complication rates resulting from radiotherapy alone were not available [34]. Lau et al. in 2013 showed predictors of complication after surgery for spinal metastases, nevertheless the study was inclusive of other solid and hematological cancer [42].

Strong clinical research publications with an algorithm for management of patients with metastatic spine disease are remarkably very few available [19]. Among the advantages of review of cases can be seen in other places. The percentage of cancer patients operated as an emergency in a spine surgery care unit had decreased significantly between 2005 and 2012. The study highlighted the need of multidisciplinary approach for management of MSCC [43]. A retrospective records reviewed in year 2012 for management of MSCC in London revealed $21 \%(n=27)$ of patients with confirmed spinal cord compression showed spinal cord compression as the first presentation of malignancy [44]. Their findings include of $67 \%$ of cases received radiotherapy as their first intervention compared with $24 \%$ who were treated initially with surgery. Their treatments patterns for MSCC over the past three years were relatively unchanged [44]. Lee et al. [19] in the review found very few Class I studies to support treatment recommendations for metastatic spine disease. In 2014, Lee et al. suggested meta-analysis with regards to treatment of direct decompressive surgical resection with radiotherapy, than radiotherapy treatment alone [18].

Clinical pathway is a form of task orientated care plans in which essential steps in care of patients with a specific clinical problem are 
detailed out and the patient's expected clinical course is described [45]. We had translated our findings into clinical pathways for managing MSCC cases in our orthopedics unit. Naturally, patients may have clinical variability upon presentation and subsequently can present with progression of the cancer. Therefore management of treatment concerned in our study was at first time of meeting the patients in wards.

There are advantages of implementing clinical pathway in a health care centre. A review of literatures from 1966 to 2007 found that the implementation of clinical pathways for surgical interventions had lead to various improvements in clinical care in surgery and usage should therefore be encouraged into routine care in surgery. The positive effects of their clinical pathways were seen in economic aspect, on the quality of treatment outcomes and patient satisfaction, and many more [46]. Clinical pathways can aid improvement of process of care. Optimizing quality of life is vital in managing MSCC as early diagnosis and expert multidisciplinary care and rehabilitation are needed. Care for MSCC is a challenging area as cancer cause significant suffering but within limited lifespan as compared to other chronic diseases. Our dedicated spine nursing care contributes to the objective of care in our centre. Others have shown that some critical components of the care pathway for 'good prognosis patients' and any inappropriate hospitalization episode resulted from management of 'poor prognosis patients' in a centre could be assessed in an audit [47]. The audit had suggested on development of clinical protocols and patient care pathways, so that appropriate clinical decisions, including appropriate surgical referral and optimal end of life care are made available to patients. Audits based on the care pathway enable organization to monitor achievement in management of MSCC against national standards benchmark. For example, audits conducted in United Kingdom following published guidance for the management of MSCC recommended by NICE [47]. With regards to economic evaluation study, the effectiveness of surgery versus non operative management in patients with MECSS based on study by Furlan et al. [48] and Thomas et al. [49] were compared by Fehlings et al. [50]. The decompressive spinal surgery followed by radiotherapy is not only more effective, but also more costly than therapeutic modality with radiotherapy alone. However, interpretation of the findings must be with caution [50].

Our study inherently limited by its retrospective design for one year information in a public tertiary health care centre. Nevertheless, we achieved our objectives where our finding descriptions were as results of practices of differences surgical skill and judgment among physicians and surgeons in a single institution. Our public funded institution is equipped with dedicated oncology unit and spine team working in a multidisciplinary approach. The clinical pathways designed are inclusive of patients with MSCC who received nonsurgical intervention including radiation therapy, and supportive care as first encounter in specialists setting.

\section{Conclusion}

MSCC event results in considerable morbidity for cancer patients and could impose an increased economic pressure on healthcare system. On the other hand, advances in diagnosis and treatment modalities with multi-disciplinary approach are improving the management of bone events in cancer. Health care system continues to change towards more patients focused of care. Nevertheless the care process should be in line with initiatives improving quality and efficiency and accessibility by healthcare providers and policy-makers. The clinical pathways designed aids authors estimating following cost incurred by the activities in managing cord compression secondary to spine metastases among breast or prostate cancers patients in public teaching hospital. The activities to manage the patients will determine the activity based costing that take place on the provider's perspectives. Burden of treatments acts as base for policy-makers to further evaluates other mode of treatments available in medical fields. Current pathways contribute to another larger pathway for managing bone events in public hospital.

\section{References}

1. Fornasier VL, Horne JG (1975) Metastases to the vertebral column. Cancer 36: 590594. [Crossref]

2. Harel R, Angelov L (2010) Spine metastases: current treatments and future directions Eur J Cancer 46: 2696-2707. [Crossref]

3. Harrington KD (1993) Metastatic tumors of the spine: diagnosis and treatment. $J \mathrm{Am}$ Acad Orthop Surg 1: 76-86. [Crossref]

4. Rose PS, Buchowski JM (2011) Metastatic disease in the thoracic and lumbar spine: evaluation and management. J Am Acad Orthop Surg 19: 37-48. [Crossref]

5. Jacobs WB, Perrin RG (2001) Evaluation and treatment of spinal metastases: an overview. Neurosurg Focus 11: e10. [Crossref]

6. Steinmetz MP, Mekhail A, Benzel EC (2001) Management of metastatic tumors of the spine: strategies and operative indications. Neurosurg Focus 11: e2. [Crossref]

7. Byrne TN (1992) Spinal cord compression from epidural metastases. $N$ Engl J Med 327: 614-619. [Crossref]

8. Klimo P Jr, Kestle JR, Schmidt MH (2003) Treatment of metastatic spinal epidura disease: a review of the literature. Neurosurg Focus 15: E1. [Crossref]

9. National Institute for Clinical Excellence's (NICE). NICE Clinical Guidance No. 75 (2008) Metastatic spinal cord compression: Diagnosis and management of adults at risk of and with metastatic spinal cord compression.

10. O'Connor MI, Currier BL (1992) Metastatic disease of the spine. Orthopedics 15: 611620. [Crossref]

11. Cole JS, Patchell RA (2008) Metastatic epidural spinal cord compression. Lancet Neurol 7: 459-466. [Crossref]

12. Heldmann U, Myschetzky PS, Thomsen HS (1997) Frequency of unexpected multifocal metastasis in patients with acute spinal cord compression. Evaluation by low-field MR imaging in cancer patients. Acta Radiol 38: 372-375. [Crossref]

13. Klimo P Jr, Schmidt MH (2004) Surgical management of spinal metastases. Oncologist 9: 188-196. [Crossref]

14. Quraishi NA, Rajagopal TS, Manoharan SR, Elsayed S, Edwards KL, et al. (2013) Effect of timing of surgery on neurological outcome and survival in metastatic spinal cord compression. Eur Spine J 22: 1383-1388. [Crossref]

15. Vanhaecht K, Panella M, Van Zelm R, Sermeus W (2010) An overview on the history and concept of care pathways as complex interventions. Int $J$ of Care Pathways 14: $117-123$

16. Akram H, Allibone J (2010) Spinal Surgery for Palliation in Malignant Spinal Cord Compression. Clin Oncol 22: 792-800. [Crossref]

17. Abrahm JL (2004) Assessment and treatment of patients with malignant spinal cord compression. J Support Oncol 2: 377-391. [Crossref]

18. Lee CH, Kwon JW, Lee J, Hyun SJ, Kim KJ, et al. (2014) Direct decompressive surgery followed by radiotherapy versus radiotherapy alone for metastatic epidural spinal cord compression: a meta-analysis. Spine (Phila Pa 1976) 39: E587-592. [Crossref]

19. Kaloostian PE, Yurter A, Zadnik PL, Sciubba DM, Gokaslan ZL (2014) Current paradigms for metastatic spinal disease: an evidence-based review. Ann Surg Oncol 21: 248-262. [Crossref]

20. Loblaw DA, Laperriere NJ (1998) Emergency treatment of malignant extradural spinal cord compression: an evidence-based guideline. J Clin Oncol 16: 1613-1624. [Crossref]

21. Ruppert LM (2013) The role of corticosteroids in the treatment of metastatic epidural spinal cord compression. J Palliat Care Med 1: 106

22. Vyvey M (2010) Steroids as pain relief adjuvants. Can Fam Physician 56: 1295-1297, e415. [Crossref] 
23. Nair C, Panikkar S, Ray A (2014) How not to miss metastatic spinal cord compression. Br J Gen Pract 64: e596-598. [Crossref]

24. Husband DJ (1998) Malignant spinal cord compression: prospective study of delays in referral and treatment. BMJ 317: 18-21. [Crossref]

25. Thirlwell C, Brock CS (2003) Emergencies in oncology. Clin Med 3: 306-310. [Crossref]

26. Freeman AK, Sumathi VP, Jeys L (2015) Metastatic tumours of bone. Surgery (Oxford) 33: 34-39.

27. Kato S, Murakami H, Minami T, Demura S, Yoshioka K, et al. (2012) Preoperative embolization significantly decreases intraoperative blood loss during palliative surgery for spinal metastasis. Orthopedics 35: e1389-e1395. [Crossref]

28. Yurter A, Ju DG, Sciubba DM (2014) Management of metastatic spine disease. JSM Neurosurg Spine 2: 1020. [Crossref]

29. Malhotra NR, Kosty J, Sanborn M, Bekisz JM, Mooncai TW, et al. (2014) Optimal approach to circumferential decompression and reconstruction for thoracic spine metastatic disease. Ann Surg Oncol 21: 2864-2872. [Crossref]

30. Phelps KD, Patt JC (2014) Diagnosis and management of patients with carcinoma metastatic to the spine. Current Orthopaedic Practice 25: 525-533.

31. Quan GM, Vital JM, Aurouer N, Obeid I, Palussière J, et al. (2011) Surgery improves pain, function and quality of life in patients with spinal metastases: a prospective study on 118 patients. Eur Spine J 20: 1970-1978. [Crossref]

32. Chaichana KL, Pendleton C, Wolinsky JP, Gokaslan ZL, Sciubba DM (2009) Vertebral compression fractures in patients presenting with metastatic epidural spinal cord compression. Neurosurgery 65: 267-274. [Crossref]

33. Liang T, Wan Y, Zou X, Peng X, Liu S (2013) Is surgery for spine metastasis reasonable in patients older than 60 years? Clin Orthop Relat Res 471: 628-639. [Crossref]

34. Kim JM, Losina E, Bono CM, Schoenfeld AJ, Collins JE, et al. (2012) Clinical outcome of metastatic spinal cord compression treated with surgical excision \pm radiation versus radiation therapy alone: a systematic review of literature. Spine (Phila Pa 1976) 37: 78-84. [Crossref]

35. Sayed MM, Abdel-Wanis ME, El-Sayed MI (2013) Single fraction compared with multiple fraction re-irradiations in patients with painful bone metastases. $J$ Cancer $\mathrm{Sci}$ Ther 5: 089-093.

36. Robson P (2014) Metastatic spinal cord compression: a rare but important complication of cancer. Clin Med 14: 542-545. [Crossref]

37. Spencer BA, Shim JJ, Hershman DL, Zacharia BE, Lim EA, et al. (2014) Metastatic epidural spinal cord compression among elderly patients with advanced prostate cancer. Support Care Cancer 22: 1549-1555. [Crossref]
38. Patchell RA, Tibbs PA, Regine WF, Payne R, Saris S, et al. (2005) Direct decompressive surgical resection in the treatment of spinal cord compression caused by metastatic cancer: a randomised trial. Lancet 366: 643-648. [Crossref]

39. Valesin Filho ES, de Abreu LC, Lima GH, de Cubero DI, Ueno FH, et al. (2013) Pain and quality of life in patients undergoing radiotherapy for spinal metastatic disease treatment. Int Arch Med 6: 6. [Crossref]

40. Zaikova O, Fossa SD, Kongsgaard U, Kvaloy S, Giercksky KE, et al. (2010) Pain after palliative radiotherapy for spine metastases. Clin Oncol (R Coll Radiol) 22: 828-836. [Crossref]

41. Lee BH, Kim TH, Chong HS, Moon ES, Park JO, et al. (2013) Prognostic factor analysis in patients with metastatic spine disease depending on surgery and conservative treatment: review of 577 cases. Ann Surg Oncol 20: 40-46. [Crossref]

42. Lau D, Leach MR, Than KD, Ziewacz J, La Marca F, et al. (2013) Independen predictors of complication following surgery for spinal metastasis. Eur Spine $J 22$ : 1402-1407. [Crossref]

43. Fahed Z, Marie-Helene V, Patrick D, Aboukais R, Louis G, et al. (2015) Management of neoplastic spinal tumors in a spine surgery care unit. Clin Neurol Neurosurg 128: 35-40. [Crossref]

44. Savage P, Sharkey R, Kua T, Schofield L, Richardson D, et al. (2014) Malignant spina cord compression: NICE guidance, improvements and challenges. QJM 107: 277-282. [Crossref]

45. Campbell H1, Hotchkiss R, Bradshaw N, Porteous M (1998) Integrated care pathways. BMJ 316: 133-137. [Crossref]

46. Ronellenfitsch U, Rössner E, Jakob J, Post S, Hohenberger P, et al. (2008) Clinica Pathways in surgery: should we introduce them into clinical routine? A review article. Langenbecks Arch Surg 393: 449-457. [Crossref]

47. McGivern UM, Drinkwater KJ, Clarke JI, Locke I (2014) A royal college of radiologists national audit of radiotherapy in the treatment of metastatic spinal cord compression and implications for the development of acute oncology services. Clin Oncol (R Coll Radiol) 26: 453-460. [Crossref]

48. Furlan JC, Chan KK, Sandoval GA, Lam KC, Klinger CA, et al. (2012) The combined use of surgery and radiotherapy to treat patients with epidural cord compression due to metastatic disease: a cost-utility analysis. Neuro Oncol 14: 631-640. [Crossref]

49. Thomas KC, Nosyk B, Fisher CG, Dvorak M, Patchell RA, et al. (2006) Costeffectiveness of surgery plus radiotherapy versus radiotherapy alone for metastatic epidural spinal cord compression. Int J Radiat Oncol Biol Phys 66: 1212-1218. [Crossref]

50. Fehlings MG, Nater A, Holmer H (2014) Cost-effectiveness of surgery in the management of metastatic epidural spinal cord compression: a systematic review. Spine (Phila Pa 1976) 39: S99-99S105. [Crossref]

Copyright: (C2015 Sharifa Ezat WP. This is an open-access article distributed under the terms of the Creative Commons Attribution License, which permits unrestricted use, distribution, and reproduction in any medium, provided the original author and source are credited. 